\title{
MULTIPLE IMAGING BY GRAVITATIONAL WAVES
}

\author{
Valerio Faraoni \\ Department of Physics and Astronomy, University of Victoria, P.O. Box 3055 \\ Victoria, B.C. Canada V8W 3P6
}

\begin{abstract}
Gravitational waves act like lenses for the light propagating through them. This phenomenon is described using the vector formalism employed for ordinary gravitational lenses, which was proved to be applicable also to a non-stationary spacetime, with the appropriate modifications. In order to have multiple imaging an approximate condition analogous to that for ordinary gravitational lenses must be satisfied. Certain astrophysical sources of gravitational waves satisfy this condition, while the gravitational wave background, on average, does not. Multiple imaging by gravitational waves is, in principle, possible, but the probability of observing such a phenomenon is extremely low.
\end{abstract}

To appear in Int. J. Mod. Phys. D 


\section{Introduction}

In general relativity (and in all metric theories of gravity) a gravitational field deflects light rays propagating through it, and gravitational waves are no exception. It is well known that exact solutions of the Einstein equations representing plane gravitational waves exhibit focusing properties on timelike and null geodesics propagating through them [1]. Light propagation through realistic linearized gravitational waves outside the laboratory has also been studied [2]-[12]. Most authors restricted their attention to cosmological gravitational waves; a particular attention was payed to the frequency shift effect induced by cosmological gravitational waves on the microwave background photons [14, 6, 13, 15], expecially after the $C O B E$ discovery of anisotropies in the cosmic microwave background. Other works focussed upon the effects of gravitational waves perturbing ordinary gravitational lenses [16] 18. In recent papers, the case of gravitational waves generated by astrophysical sources was studied [19-22 and, in some cases, optimistic statements were made about the detectability of these waves via the effects induced by their interaction with the light coming from distant objects.

The study of gravitational lensing by mass concentrations perturbing the background curvature of the universe has become a very active field of astronomy [23]. In extreme conditions multiple images of a celestial object are created, which are accompanied by high amplification events. In these situations spectacular phenomena are observed by the astronomers: multiple quasars, giant arcs, arclets and radio rings [23]. It is therefore natural to ask whether gravitational waves can create multiple images of a celestial object and give rise to the associated high amplification events. This question was posed in Refs. 24-27. The possibility of multiple imaging by gravitational waves in general relativity constitutes the subject of the present paper. Some of the ideas exposed here were anticipated in a brief abstract [27]. While the idea of multiple imaging by gravitational waves was only qualitatively sketched in Ref. [24], in Ref. [25] the Raychaudhuri equation was used to deduce that the amplification of a light beam induced by gravitational waves is of second order in the wave amplitudes, and therefore completely negligible in any situation of astrophysical interest. This conclusion is valid only when a single image of the light source is created, and cannot be extended to the case of multiple images and the associated high amplification events because the scalar formalism is known to fail in these situations 28]. Nowadays, we have formalisms that are more suitable than the Raychaudhuri equation for the description of multiple images in gravitational

lensing [23. There is, however, a problem with the available formalisms, i.e. they were introduced to describe lensing in a (conformally) static spacetime and, in principle, their validity is restricted to this class of spacetimes. Instead, the case considered in this paper 
involves a highly non-(conformally) stationary spacetime. This problem was resolved in a previous paper [29], where it was proved that the vector formalism [23] can still be applied to the case of lensing by gravitational waves, provided that a new formula for the deflection angle is calculated. This result may look intuitive, but the proof of its validity is non-trivial and requires a generalization of the Fermat principle to the case of non-stationary spacetimes, which was given only recently 30.

Using the results of Ref. [29, we approach the problem employing the vector formalism and describing the effect of the lens with a plane-to-plane map. The vanishing of the Jacobian of this geometric map is associated with the failure of the inverse map, and defines critical lines and caustics. An approximate condition for multiple imaging is derived, and it is shown to be analogous to the well known approximate condition for multiple imaging by ordinary gravitational lenses [31]. Order of magnitude estimates show that the condition for the creation of multiple images by gravitational waves is satisfied by certain astrophysical sources of waves considered in the literature. This happens thanks to the balance between the (large) source-lens distances and the (small) wave amplitudes, and because the lensing waves in the sky can have relatively large amplitudes (i.e. larger than those expected in the Solar System). On average, the gravitational wave background is not capable of multiple imaging. It is concluded that multiple imaging by gravitational waves is, in principle, possible, but the probability of observing such a phenomenon is very low.

Although various aspects of lensing could be considered, in this paper we focus on the creation of multiple images because the associated high amplification events are the most dramatic effects, and the most relevant for the detection of lensing events and, indirectly, of gravitational waves.

The plan of the paper is as follows: in Sec. 2 and Sec. 3 we introduce the necessary tools and the assumptions underlying this work. Emphasis is given to the computation of the deflection angle (Sec. 2) and to the validity of the thin lens approximation (Sec. 3). In Sec. 4, the modified vector formalism is applied to lensing gravitational waves and an approximate condition for the creation of multiple images is derived. In Sec. 5, gravitational waves and ordinary gravitational lenses are compared, while Sec. 6 explores the possibility that the condition for multiple imaging is satisfied by astrophysical sources of gravitational waves. Sec. 7 contains final remarks.

We use units in which the Newton constant $G$ and the speed of light $c$ assume the value unity (but $G$ and $c$ will occasionally be restored). The metric signature is +2 ; Latin indices run from 1 to 3, and Greek indices run from 0 to 3. A comma denotes partial differentiation. 


\section{Computation of the deflection angle}

Let us consider gravitational waves localized in a region of space between a light source and an observer. The spacetime metric is given, in an asymptotically Cartesian coordinate system $\{t, x, y, z\}$, by

$$
g_{\mu \nu}=\eta_{\mu \nu}+h_{\mu \nu},
$$

where $\eta_{\mu \nu}=\operatorname{diag}(-1,1,1,1)$ and $\left|h_{\mu \nu}\right| \ll 1$ in these coordinates. Let us consider a light ray whose unperturbed path is parallel to the $z$-axis. The photon describing this ray has four-momentum

$$
p^{\mu}=p_{(0)}^{\mu}+\delta p^{\mu}=\left(1+\delta p^{0}, \delta p^{1}, \delta p^{2}, 1+\delta p^{3}\right),
$$

where $\delta p^{\mu}$ are small deflections (of order $h_{\mu \nu}$ ) and the unperturbed photon four-momentum is $p_{(0)}^{\mu}=(1,0,0,1)$. The computations are performed in the geometric optics approximation, that holds if the wavelength $\lambda_{g w}$ of the gravitational wave is much larger than the photon wavelength $\lambda_{e m}$, and if

$$
\lambda_{e m}>\lambda_{g w}^{2} / D_{L},
$$

where $D_{L}$ is the observer-lens distance. Equation (2.3) guarantees that the size of the interference fringes which eventually form at the observer's position is not comparable to the "geometrical shadow" of the lens [25]. In order to make computations to second order in the wave amplitudes, tensor indices are lowered and raised with $g_{\mu \nu}$ and with

$$
g^{\mu \nu}=\eta^{\mu \nu}-h^{\mu \nu}+O\left(h^{2}\right) \equiv \eta^{\mu \nu}-\eta^{\mu \rho} \eta^{\nu \sigma} h_{\rho \sigma}+O\left(h^{2}\right),
$$

respectively. The symbols $O(h), O\left(h^{2}\right)$ denote the orders of magnitude of $h_{\mu \nu}$ and of terms quadratic in $h_{\mu \nu}$, respectively. The equation of null geodesics gives

$$
\frac{d\left(\delta p^{\mu}\right)}{d \lambda}+\Gamma_{\rho \sigma}^{\mu}\left(p_{(0)}^{\rho}+\delta p^{\rho}\right)\left(p_{(0)}^{\sigma}+\delta p^{\sigma}\right)=0
$$

where $\lambda$ is an affine parameter along the null geodesics and

$$
\Gamma_{\rho \sigma}^{\mu}=\frac{1}{2} g^{\mu \nu}\left(h_{\nu \rho, \sigma}+h_{\nu \sigma, \rho}-h_{\rho \sigma, \nu}\right)
$$

One obtains

$$
\frac{d\left(\delta p^{\mu}\right)}{d \lambda}+\eta^{\mu \nu}\left(h_{\nu 0,0}+h_{\nu 0,3}+h_{\nu 3,0}+h_{\nu 3,3}-h_{03, \nu}-\frac{1}{2} h_{00, \nu}-\frac{1}{2} h_{33, \nu}\right)+\mathrm{O}\left(h^{2}\right)=0 .
$$


We introduce the notation

$$
A_{\lambda} \equiv p^{\alpha} A_{\alpha} ; \quad B_{, \lambda} \equiv p^{\alpha} \frac{\partial B}{\partial x^{\alpha}} .
$$

Using the expression

$$
\Gamma_{\lambda \lambda}^{\mu}=\eta^{\mu \nu}\left(h_{\nu \lambda, \lambda}-\frac{1}{2} h_{\lambda \lambda, \nu}\right)+O\left(h^{2}\right)
$$

and Eq. (2.7), one obtains

$$
\delta p^{\mu}=-\int_{S}^{O} d \lambda\left(h_{\lambda, \lambda}^{\mu}-\frac{1}{2} h_{\lambda \lambda}{ }^{\mu}{ }^{\mu}\right)+O\left(h^{2}\right),
$$

where the indices are now raised with $\eta^{\mu \nu}$ and the integral is computed along the photon path from the source to the observer. Since a localized pulse of gravitational waves is considered, the first term in the integrand gives a vanishing boundary term円 and it follows that

$$
\delta p^{\mu}=\frac{1}{2} \int_{S}^{O} d \lambda\left(h_{00}+2 h_{03}+h_{33}\right)^{, \mu}+O\left(h^{2}\right) .
$$

Performing the integration along the unperturbed photon path instead of the actual path involves only an error of order $h^{2}$ and hence the first order result is given by

$$
\delta p_{(1)}^{\mu}=\frac{1}{2} \int_{S}^{O} d z\left(h_{00}+2 h_{03}+h_{33}\right)^{, \mu}
$$

(notice that the deflection angle $\delta p^{\mu}$ is the opposite of the angle used in the theory of ordinary gravitational lenses, in which deflection from a straight line is defined as having the opposite orientation). For pulses of gravitational waves this integral is extended to a region of space of order $P$ (the characteristic period of the waves) where the pulse is localized so that, in order of magnitude, $\delta p \sim P \cdot h / P=h$. If the gravitational wave background is considered, it might appear that the computation of the integral along the whole photon path from the source to the observer gives $\delta p \sim D h / P$. However, this secular effect is absent, i.e. the deflection does not cumulate with the travelled distance, due to the transversality of gravitational waves and to the equality between the speed of light and that of the random inhomogeneities of the medium (the gravitational waves), as shown in Refs. [2, 14, 6, 8, 11, 12].

\footnotetext{
${ }^{1}$ The boundary term is usually negligible [22]. The case of "pulses with memory" was considered in Ref. [20].
} 
We also compute the second order correction $\delta p_{(2)}^{\mu}$ to the deflection, which was not given in previous references and will be used in Sec. $4 . \delta p_{(2)}^{\mu}$ is obtained by inserting Eq. (2.12) into the equation of null geodesics

$$
\frac{d\left(\delta p^{\mu}\right)}{d \lambda}+\Gamma_{\lambda \lambda}^{\mu}+2 \Gamma_{\lambda \sigma}^{\mu} \delta p_{(1)}^{\sigma}+O\left(h^{3}\right)=0
$$

where $\delta p^{\mu}=\delta p_{(1)}^{\mu}+\delta p_{(2)}^{\mu}$, which gives

$$
\begin{gathered}
\delta p_{(2)}^{\mu}=\int_{S}^{O} d z\left[-\left(\partial_{\sigma} h_{0}^{\mu}+\partial_{\sigma} h_{3}^{\mu}+\partial_{0} h_{\sigma}^{\mu}+\partial_{3} h_{\sigma}^{\mu}-\partial^{\mu} h_{0 \sigma}-\partial^{\mu} h_{3 \sigma}\right) \delta p_{(1)}^{\sigma}\right. \\
\left.+h^{\mu \alpha}\left(h_{\alpha 0,0}+h_{\alpha 0,3}+h_{\alpha 3,0}+h_{\alpha 3,3}-h_{03, \alpha}-\frac{1}{2} h_{00, \alpha}-\frac{1}{2} h_{33, \alpha}\right)\right]+O\left(h^{3}\right),
\end{gathered}
$$

with $\delta p_{(1)}^{\sigma}$ given by Eq. (2.12) and where the integration is performed again along the unperturbed photon's path instead of the actual path, the difference contributing only by a third order term. Equation (2.14) allows the computation of the second order contribution to the divergence $\partial_{A}\left(\delta p^{A}\right)$ (the first order contribution being zero [25]):

$$
\begin{aligned}
& \partial_{A}\left(\delta p^{A}\right)=\int_{S}^{O} d z\left\{-\delta p_{(1)}^{\sigma}\left(h_{0, \sigma A}^{A}+h_{3, \sigma A}^{A}+h_{\sigma, 0 A}^{A}+h_{\sigma, 3 A}^{A}\right)\right. \\
& -\partial_{A}\left(\delta p_{(1)}^{\sigma}\right)\left(h_{0, \sigma}^{A}+h_{3, \sigma}^{A}+h_{\sigma, 0}^{A}+h_{\sigma, 3}^{A}-h_{0 \sigma}{ }^{A}{ }^{A}-h_{3 \sigma}{ }^{A}\right) \\
& +h_{, A}^{A \alpha}\left(h_{\alpha 0,0}+h_{\alpha 0,3}+h_{\alpha 3,0}+h_{\alpha 3,3}-h_{03, \alpha}-\frac{1}{2} h_{00, \alpha}-\frac{1}{2} h_{33, \alpha}\right) \\
& \left.+h^{A \alpha}\left(h_{\alpha 0,0 A}+h_{\alpha 0,3 A}+h_{\alpha 3,0 A}+h_{\alpha 3,3 A}-h_{03, \alpha A}-\frac{1}{2} h_{00, \alpha A}-\frac{1}{2} h_{33, \alpha A}\right)\right\} \text {, }
\end{aligned}
$$

where $A=1,2$.

\section{The thin lens approximation}

In the next section we will apply the vector formalism for gravitational lenses, with the appropriate modifications, to the case of lensing by gravitational waves. One of the assumptions underlying the vector formalism is the validity of the thin lens approximation, i.e. that the size of the lens be negligible in comparison to the length of the path

travelled by the photon. This approximation requires special care in the case of lensing gravitational waves, and is dealt with in what follows. 
To be specific, we restrict to the case of gravitational waves generated by astrophysical sources and propagating with wavefronts that are approximately spherical at a sufficiently large distance from the source. In this situation, a photon coming from infinity and entering the gravitational wave will never leave it, hence the thin lens approximation might seem to be inapplicable. However, the photon is appreciably deflected only during a small fraction of the time spent inside the gravitational wave, at the minimum distance from the source of gravitational waves (where the wave amplitude is maximum). The region of space in which the photon is appreciably deflected has a size that is much smaller than the total length of the path travelled by the photon. In fact, let $h \sim h_{S} R_{S} / r$ be the order of magnitude of the gravitational wave amplitude at a distance $r$ from the source of waves, where $R_{S}$ and $h_{S}$ are, respectively, the amplitude at the source and the Schwarzschild radius of the object generating gravitational waves. One has $h_{S} \simeq \epsilon M / R$, where $\epsilon, M$ and $R$ are the efficiency of the generation process, the mass and radius of the object, respectively. For neutron stars, $M / R \sim 1 / 20$, and $\epsilon \sim 0.1$ is a very generous estimate of the efficiency (see Sec. 6). These numbers give a deflection angle $\delta \sim h \sim 5 \cdot 10^{-3}(1 \mathrm{~km} / r)$. The minimum detectable separation between multiple images is of the order $\delta_{0} \simeq 10^{-4}$ arcseconds, marginally accessible by VLBI. The requirement $\delta \geq \delta_{0}$ gives $r \sim h_{S} R_{S} / \delta \leq 3 \cdot 10^{7} \mathrm{~km}$. This distance is very small in comparison with the path travelled by the photon, usually of the order of many megaparsecs. Allowing a separation between the images of a light source smaller than $\delta_{0} \sim 10^{-4}$ arcseconds would still give high amplification events although it would be impossible to resolve the multiple images (this occurs, e.g. in microlensing by stars or compact objects [23]). Even in this case the size of the region where the deflection takes place is negligible in comparison to the size of the photon path.

A second argument supporting the validity of the thin lens approximation is the following: Due to the transversality of gravitational waves, a photon is not deflected by a gravitational wave propagating parallely to the photon path. To be more specific, let $\vec{k}$ be the (3-dimensional) wave vector of a gravitational wave of finite (short) duration. If the component $k^{3}$ is zero (i.e. the gravitational wave propagates orthogonally to the unperturbed photon path), it is clear that the gravitational wave is confined to a plane and that the thin lens approximation holds. One may conjecture that this approximation fails to be satisfied as $\left|k^{3}\right|$ increases, but this would be incorrect. We give an explicit proof for a single monochromatic gravitational wave. In the extreme case $|\vec{k}|=\left|k^{3}\right|$ (gravitational wave antiparallel[ to the photon path) the light rays are not deflected,

\footnotetext{
${ }^{2}$ If the gravitational wave propagates parallely to the light rays, the light never reaches the gravitational wave, due to the equality between the speed of gravitational waves $c_{g w}$ and the speed of light.
} 
due to the transversality of gravitational waves. This fact can be deduced by Refs. [2, 11] or by Eq. (2.12). In fact, the use of the transverse-traceless (TT) gauge in Eq. (2.12) gives $h_{00}^{(T T)}=h_{03}^{(T T)}=h_{33}^{(T T)}=0$, which implies $\delta p^{A}=0$. The vanishing of the deflection angle (a gauge-invariant quantity) in the TT gauge implies its vanishing in any gauge.

If the direction of propagation of the gravitational wave and the (unperturbed) photon path are not parallel nor orthogonal, the light rays can be approximated by zig-zag paths with (3-dimensional) unit tangent vector $\vec{n}$, and the deflection depends on the quantity $\left(h_{00}+2 h_{0 i} n^{i}+h_{i j} n^{i} n^{j}\right)$, which replaces the argument of the integral in Eq. (2.12) [29]. The vector $\vec{n}$ is decomposed into its components parallel and perpendicular to $\vec{k}$ :

$$
\vec{n}=\vec{n}_{\|}+\vec{n}_{\perp}
$$

where $\vec{n}_{\|}=\vec{k}\left(\vec{n} \cdot \vec{k} / k^{2}\right)$ and $\vec{n}_{\perp} \cdot \vec{k}=0$. By introducing the quantities

$$
\begin{gathered}
h_{0 \|} \equiv h_{0 i} n_{\|}^{i} \quad, \quad h_{0 \perp} \equiv h_{0 i} n_{\perp}^{i}, \\
h_{\|\|} \equiv h_{i j} n_{\|}^{i} n_{\|}^{j} \quad, \quad h_{\| \perp} \equiv h_{i j} n_{\|}^{i} n_{\perp}^{j} \quad, \quad h_{\perp \perp} \equiv h_{i j} n_{\perp}^{i} n_{\perp}^{j},
\end{gathered}
$$

one can write

$$
h_{00}+2 h_{0 i} n^{i}+h_{i j} n^{i} n^{j}=h_{00}+2\left(h_{0 \|}+h_{0 \perp}+h_{\perp \|}\right)+h_{\perp \perp}+h_{\|\|} .
$$

The monochromatic gravitational wave is best described in the TT gauge in which $h_{0 \mu}=h_{\|\|}=h_{\| \perp}=0$. In this gauge

$$
h_{00}+2\left(h_{0 \|}+h_{0 \perp}+h_{\perp \|}\right)+h_{\perp \perp}+h_{\|\|}=h_{\perp \perp} .
$$

Therefore, the "component" of the gravitational wave propagating parallely to the (unperturbed) photon path does not affect the light rays, and the thin lens approximation is valid.

\section{The vector formalism and the occurrence of mul- tiple images}

The vector formalism familiar from the description of ordinary gravitational lenses can

be employed also for the case in which the lens is a gravitational wave, provided that the

This situation may change in alternative theories of gravity in which $c_{g w} \neq c[50$. 
deflection angle (2.12) is used. The proof of this statement was presented in a previous paper [29], and relies on a rigorous formulation of the Fermat principle valid for arbitrary (non-stationary) spacetimes which became available only recently [30].

We set the geometry of the problem as customary in gravitational lens theory: we consider only waves which are localized near a plane $z=z_{w}$ to satisfy the thin lens approximation; the deflection takes place in this plane (lens or image plane in the usual language) [23]. This description is clearly adequate for the case of a gravitational wave propagating in a direction orthogonal to the $z$-axis. Naively, one might expect that the description becomes less and less correct as the direction of propagation of the gravitational wave becomes closer and closer to the $z$-axis, but this is not the case, as discussed in the previous section.

Let $\underline{x}=(x, y)$ be the apparent source position in the lens plane, and $\underline{s}=\left(s_{x}, s_{y}\right)$ be the true source position (i.e. its position were the lensing wave absent). The plane orthogonal to the $z$-axis and passing through the source is referred to as the source plane. The true source position in the lens plane is related to that in the source plane by identifying the two planes). The deflection is described by a two-dimensional vector field $\delta p^{A}(\underline{x})(A=1,2)$ in the lens plane. The action of the lens is described by a plane-to-plane mapping $x^{A} \longmapsto s^{A}$, where $\underline{s}$ is given by the lens equation

$$
s^{A}=x^{A}+\frac{D_{L} D_{L S}}{D_{S}} \delta p^{A}(\underline{x})
$$

and where $D_{L}, D_{L S}$ and $D_{S}$ are the observer-lens, lens-source, and observer-source distances, respectively. As customary in gravitational lens theory, we can fit cosmology into the model by assuming the $D$ 's to be angular diameter distances in a FriedmannLemaitre-Robertson-Walker (FLRW) universe [23]. However, for the sake of simplicity, we assume that the background is flat and that the $D$ 's denote Euclidean distances, with $D_{S}=D_{L}+D_{L S}$. As described in Ref. [29], the use of the Fermat principle generalized to non-stationary spacetimes [30 allows one to derive the lens equation (4.1), with the deflection angle $\delta p^{A}$ given by Eq. (2.12).

The map described by Eq. (4.1) has the Jacobian matrix

$$
J\left(\begin{array}{l}
\underline{s} \\
\underline{x}
\end{array}\right)=\left(\frac{\partial s^{A}}{\partial x^{B}}\right)=\left(\begin{array}{cc}
1+D \partial_{x}\left(\delta p^{x}\right) & D \partial_{y}\left(\delta p^{x}\right) \\
D \partial_{x}\left(\delta p^{y}\right) & 1+D \partial_{y}\left(\delta p^{y}\right)
\end{array}\right),
$$

where $D \equiv D_{L} D_{L S} / D_{S}$. The inverse matrix $A=J^{-1}$ represents the amplification tensor, while its determinant $\mathcal{A}=\operatorname{Det}(J)^{-1}$ is the (scalar) amplification. Since the 
surface brightness is conserved during lensing whenever geometric optics holds [32] and

$$
\mathcal{A}=\frac{\text { area of an infinitesimal region in the lens plane }}{\text { area of the corresponding region in the source plane }},
$$

$\mathcal{A}$ has also the meaning of the ratio of light intensities with and without the lens [23]. A small circular source will be imaged into a small ellipse whose eccentricity $e$ is given by the ratio of the eigenvalues $e_{ \pm}$of $A$ :

$$
\left(1-e^{2}\right)^{1 / 2}=\left|\frac{e_{+}}{e_{-}}\right| .
$$

The vanishing of the Jacobian $\operatorname{Det}(J)$ indicates the failure of invertibility of the map (4.1). The loci of points in the lens plane where $\operatorname{Det}(J)=0$ are called critical lines and the corresponding curves in the source plane are called caustics. Critical lines [caustics] separate regions corresponding to different numbers of images in the lens [source] plane. Therefore, the occurrence of multiple images is signalled by the vanishing of $\operatorname{Det}(J)$, and this is the condition that we will study in the following. These concepts are familiar from standard gravitational lens theory [23]. We now proceed to study the features that are peculiar to lensing gravitational waves.

Let us consider the Jacobian determinant

$$
\operatorname{Det}(J)=1+D \frac{\partial\left(\delta p^{A}\right)}{\partial x^{A}}-D^{2}\left[\partial_{y}\left(\delta p^{x}\right) \cdot \partial_{x}\left(\delta p^{y}\right)-\partial_{x}\left(\delta p^{x}\right) \cdot \partial_{y}\left(\delta p^{y}\right)\right] ;
$$

the divergence $\partial\left(\delta p^{A}\right) / \partial x^{A}$ vanishes to first order. This follows from the fact that it represents the expansion scalar of a congruence of null rays. It was proved correctly in Ref. [25] that this quantity is of second order in the wave amplitudes; we give an independent proof in the Appendix. Eq. (4.5) can be written as follows

$$
\begin{gathered}
\operatorname{Det}(J)=1+J_{1}+J_{2}, \\
J_{1}=\sqrt{f(\alpha)} D_{S} \frac{\partial\left(\delta p^{A}\right)}{\partial x^{A}}, \\
J_{2}=f(\alpha) D_{S}^{2}\left[\partial_{x}\left(\delta p^{x}\right) \cdot \partial_{y}\left(\delta p^{y}\right)-\partial_{y}\left(\delta p^{x}\right) \cdot \partial_{x}\left(\delta p^{y}\right)\right],
\end{gathered}
$$

where $\alpha \equiv D_{L S} / D_{S}$ and $f(\alpha)=\alpha^{2}(1-\alpha)^{2}$. The polynomial $f(\alpha)$ is symmetric about $\alpha=1 / 2$ (corresponding to $D_{L}=D_{L S}$ ), where it assumes its maximum value $1 / 16 \simeq$ 0.0625 . 
From Eq. (2.15) one obtains the order of magnitude estimate

$$
J_{1} \sim D \partial_{A}\left(\delta p^{A}\right) \sim \frac{D}{P} h^{2}
$$

Since $J_{2} \sim(D / P)^{2} h^{2}$, we neglect $J_{1}$ in comparison to $J_{2}$ in the Jacobian determinant of Eq. (4.5) when large values of $D / P$ are considered, which is the case of interest.

In order for $\operatorname{Det}(J)$ to vanish (i.e. to have multiple images of the light source), it must be $J_{1}+J_{2}<0$. Since $J_{1}$ and $J_{2}$ are terms of order $h^{2}$ times $D / P$ or $(D / P)^{2}$, respectively (see Eqs. (2.12) and (4.9)), in order to have $\operatorname{Det}(J)=0$, a large value of the distance $D$ must balance for the small values of the wave amplitudes. Moreover, for large values of $D / P, J_{1}$ is negligible in comparison with $J_{2}$, and hence in order to have multiple imaging it must be

$$
f(\alpha)\left[D_{S} \partial_{A}\left(\delta p^{B}\right)\right]^{2} \sim 1
$$

For standard gravitational lenses, the probability of lensing of a distant source is approximately maximum when the lens is halfway between the source and the observer [33. This applies also to lensing gravitational waves and therefore values of the numerical factor $f(\alpha)$ far from its maximum are not statistically significant. We take $f(\alpha)$ in the

range $\frac{1}{100}-\frac{1}{16}$ near its maximum. Then, in order to have multiple imaging, the following condition must be satisfied:

$$
D \frac{\delta p}{P} \sim \frac{D h}{P} \geq 4-10
$$

where $P$ is the period of the gravitational wave (computed at the redshift of the lens plane, if the $D^{\prime} s$ are chosen to represent angular diameter distances in a FLRW universe) and where we used $\delta p \sim h$ (see Eq. (2.12)). The inequality (4.11) can be written in the form

$$
\frac{h}{P} \geq \mathcal{S}_{c}
$$

where $\mathcal{S}_{c} \equiv(4-10) c / D$. The approximate condition for multiple imaging (4.12) involves the "strength" $h$ and the "size" $P$ of gravitational waves, the geometry of the problem (through $D$ ) and the fundamental constant $c$. (4.12) is analogous to the well known condition for multiple imaging by ordinary gravitational lenses [31]

$$
\Sigma \geq \Sigma_{c} \equiv \frac{c^{2}}{4 \pi G D}
$$


where $\Sigma$ is the two-dimensional (projected or surface) density of the lens and the critical density $\Sigma_{c}$ depends only from the geometrical factor $D$ and from fundamental constants. This is a condition on the "strength" (the mass) and the size of the lens.

The energy and momentum of high frequency gravitational waves are given by the Isaacson effective stress tensor which, in the TT gauge, assumes the form

$$
T_{\mu \nu}=\frac{c^{4}}{32 \pi G}\left\langle\sum_{i, j} h_{i j, \mu}^{(T T)} h_{i j, \nu}^{(T T)}\right\rangle
$$

where \langle\rangle denotes the Brill-Hartle average. For $\mu=\nu=0, T_{00}=\rho c^{2}$, where $\rho$ is the effective mass density of the gravitational waves. It is clear from this expression that the ratio $h / P$ is a measure of the "mass density" of the particular lens under consideration's. We comment on the analogy between eq. (4.12) and eq. (4.13): By squaring, dividing by $G$ and integrating eq. (4.12), it is deduced that

$$
\Sigma_{g w} \equiv \frac{1}{G} \int_{S}^{O} d z\left(\frac{h}{P}\right)^{2} \geq S_{c}{ }^{2} \frac{D_{S}}{G} \simeq(16-100) \frac{c^{2}}{G D} .
$$

Strictly speaking, it is eq. (4.15), not (4.12) that is the analogous of eq. (4.13): the quantity $\Sigma_{g w}$ has the dimensions of a surface density, and $S_{c}^{2} D_{S} / G$ is the critical surface density.

The particular lens under consideration always produces an odd number of images. In fact, the odd image number theorem [35] holds, its proof requiring only boundedness, smoothness and transparency of the lens (conditions satisfied by localized gravitational waves).

\section{Comparison between a gravitational wave and an ordinary gravitational lens}

It is instructive to compare the action of a gravitational wave with that of an ordinary gravitational lens. The latter is a mass distribution described by a Newtonian potential $\Phi$ (satisfying the Poisson equation $\nabla^{2} \Phi=4 \pi \rho$, where $\rho$ is the lens mass density). It is assumed that the lens is smooth, bounded and stationary, i.e

\footnotetext{
${ }^{3}$ Although the form of the perturbations $h_{\mu \nu}$ depends on the chosen gauge, its order of magnitude, and that of $h / P$, is unaffected by gauge transformations [34]. Therefore the approximate condition (4.12) for multiple imaging is gauge-independent.
} 
- $\Phi$ is continuous with its first and second derivatives;

- $\Phi \rightarrow 0$ and $\nabla \Phi \rightarrow 0$ as $r \equiv\left(x^{2}+y^{2}+z^{2}\right)^{1 / 2} \rightarrow+\infty$;

- $\partial \Phi / \partial t \simeq 0$.

The plane-to-plane map describing the lens action is given by the lens equation and the Jacobian matrix can be written [28] as

$$
J=\left(\begin{array}{cc}
1-\chi-\Lambda & -\mu \\
-\mu & 1-\chi+\Lambda
\end{array}\right),
$$

with

$$
\begin{aligned}
\chi & \equiv \frac{\Sigma}{\Sigma_{c}} \\
\Lambda & \equiv \frac{D}{c^{2}} \int_{-\infty}^{+\infty} d l\left(\frac{\partial^{2} \Phi}{\partial x^{2}}-\frac{\partial^{2} \Phi}{\partial y^{2}}\right), \\
\mu & \equiv \frac{D}{c^{2}} \int_{-\infty}^{+\infty} d l \frac{\partial^{2} \Phi}{\partial x \partial y},
\end{aligned}
$$

where $D$ and $\Sigma_{c}$ have been defined in the previous section and

$$
\Sigma \equiv \int_{-\infty}^{+\infty} d l \rho
$$

The Jacobian determinant is given by

$$
\operatorname{Det}(J)=(1-\chi)^{2}-\left(\Lambda^{2}+\mu^{2}\right) .
$$

The convergence $\chi$ describes the action of matter, while $\Lambda$ and $\mu$ describe the action of shear. For a lensing gravitational wave one obtains

$$
J_{g w}=\left(\begin{array}{cc}
1-\Lambda_{1} & -\mu_{1} \\
-\mu_{2} & 1-\Lambda_{2}
\end{array}\right),
$$

where

$$
\begin{gathered}
\Lambda_{1}=-D \partial_{x}\left(\delta p^{x}\right)=-D \partial_{x}\left(\delta p_{(1)}^{x}+\delta p_{(2)}^{x}\right) \equiv \Lambda_{g w}+\delta \Lambda_{1} \\
\Lambda_{2}=-D \partial_{y}\left(\delta p^{y}\right)=-D \partial_{y}\left(\delta p_{(1)}^{y}+\delta p_{(2)}^{y}\right) \equiv-\Lambda_{g w}+\delta \Lambda_{2}
\end{gathered}
$$




$$
\begin{aligned}
& \mu_{1}=-D \partial_{y}\left(\delta p^{x}\right)=-D \partial_{y}\left(\delta p_{(1)}^{x}+\delta p_{(2)}^{x}\right) \equiv \mu_{g w}+\delta \mu_{1} \\
& \mu_{2}=-D \partial_{x}\left(\delta p^{y}\right)=-D \partial_{x}\left(\delta p_{(1)}^{y}+\delta p_{(2)}^{y}\right) \equiv \mu_{g w}+\delta \mu_{2}
\end{aligned}
$$

To first order, one has

$$
\begin{aligned}
\Lambda_{1} & \equiv-\frac{D}{2} \int_{S}^{O} d \lambda h_{\lambda \lambda}{ }^{, x}{ }_{, x}, \\
\Lambda_{2} & \equiv-\frac{D}{2} \int_{S}^{O} d \lambda h_{\lambda \lambda}{ }^{, y}{ }_{, y}, \\
\mu_{1} & \equiv-\frac{D}{2} \int_{S}^{O} d \lambda h_{\lambda \lambda}{ }^{, x}{ }_{, y}, \\
\mu_{2} & \equiv-\frac{D}{2} \int_{S}^{O} d \lambda h_{\lambda \lambda}{ }^{, y}{ }_{, x},
\end{aligned}
$$

where the integrals are computed along the photon's path from the source to the observer. To first order, $\Lambda_{2}=-\Lambda_{1} \equiv-\Lambda_{g w}$ and $\mu_{1}=\mu_{2} \equiv \mu_{g w}$. In fact, from Eqs. (5.12)-(5.15) and from $\partial_{A}\left(\delta p^{A}\right)=0$ (see the Appendix), it follows that

$$
\begin{aligned}
\Lambda_{1}+\Lambda_{2} & =-\frac{D}{2} \int_{S}^{O} d \lambda\left(h_{\lambda \lambda}{ }^{, x}{ }_{, x}+h_{\lambda \lambda}{ }^{, y}{ }_{, y}\right)+O\left(h^{2}\right)= \\
= & -\frac{D}{2} \int_{S}^{O} d \lambda p^{\alpha} p^{\beta}\left(h_{\alpha \beta}{ }^{, x}{ }_{, x}+h_{\alpha \beta}{ }^{, y}{ }_{, y}\right)+O\left(h^{2}\right)=0+O\left(h^{2}\right),
\end{aligned}
$$

hence $\Lambda_{2}=-\Lambda_{1}+O\left(h^{2}\right)$.

In the first order approximation, tensor indices are raised and lowered with $\eta^{\mu \nu}$ and $\eta_{\mu \nu}$, respectively, and $\eta^{A B}=\eta_{A B}=\delta_{A B}$ for $A, B=1,2$. Hence, to this order, $h_{\mu \nu}{ }^{, A}{ }_{, B}=h_{\mu \nu}{ }^{B}{ }_{, A}$ and $\mu_{1}=\mu_{2}+O\left(h^{2}\right)$.

The corrections

$$
\begin{aligned}
& \delta \Lambda_{1}=-D \partial_{x}\left(\delta p_{(2)}^{x}\right), \\
& \delta \Lambda_{2}=-D \partial_{y}\left(\delta p_{(2)}^{y}\right), \\
& \delta \mu_{1}=-D \partial_{y}\left(\delta p_{(2)}^{x}\right), \\
& \delta \mu_{2}=-D \partial_{x}\left(\delta p_{(2)}^{y}\right),
\end{aligned}
$$

where $\delta p_{(2)}^{A}$ is given by Eq. (2.14), are of second order in $h$. To lowest order, we have

$$
J_{g w}=\left(\begin{array}{cc}
1-\Lambda_{g w} & -\mu_{g w} \\
-\mu_{g w} & 1+\Lambda_{g w}
\end{array}\right) .
$$


The convergence term is absent and hence the lens action is due only to the shear. This result was derived in Ref. 25] using the Raychaudhuri equation and the optical scalars formalism, and is now recovered in the vector formalism.

When the determinant of $J_{g w}$ is considered, one has

$$
\operatorname{Det}\left(J_{g w}\right)=1-\left(\Lambda_{g w}^{2}+\mu_{g w}^{2}\right)+D\left[\partial_{x}\left(\delta p_{(2)}^{x}\right)+\partial_{y}\left(\delta p_{(2)}^{y}\right)\right] .
$$

An order of magnitude estimate as outlined in Sec. 4 gives $\Lambda_{g w}^{2}, \mu_{g w}^{2} \sim(D / P)^{2} h^{2}>>$ $D \partial_{A}\left(\delta p^{A}\right) \sim(D / P) h^{2}$ for large $D / P$. Therefore, we can write

$$
\operatorname{Det}\left(J_{g w}\right)=1-\left(\Lambda_{g w}^{2}+\mu_{g w}^{2}\right)
$$

(where the term in brackets is of second order in $h D / P$ ).

It is to be noted that the deflection angle (2.11) does not depend on the frequency of the light. Gravitational waves are achromatic lenses, like ordinary gravitational lenses. However, while the latters do not shift the frequency of the photons propagating through them, lensing gravitational waves do. The effect is of first order in the wave amplitude and has been studied in detail by many authors [2, 6, 7, 9, 12, 14, expecially in conjunction with the microwave background anisotropies discovered by the COBE experiment. In addition, gravitational waves do not rotate the polarization plane of the electromagnetic field, to first order [10]. In this aspect, they behave like ordinary gravitational lenses.

It is expected that the images of a distant source created by a gravitational wave vary on timescales of the order of the wave period. This could possibly be used to explain the variability of some active galactic nuclei or active galaxies. Moreover, the details of the images configuration depend on the detailed form and parameters of the lensing wave, such as its spatial and time profile, its duration, direction of propagation and polarization (see [19] for an example).

\section{Order of magnitude estimates}

In order to apply the previous theory and the multiple images to be detectable, the following conditions must be satified:

1. geometric optics holds; 
2. the scale of separation between different images must not be smaller than $10^{-3}$ arcseconds. In fact, structures on scales $\sim 10^{-3}$ arcseconds can be resolved with VLBI, while VLA and optical techniques apply on larger scalest;

3. the lens must not be exceptionally rare, i.e. the rate of occurrence of the event generating the lensing wave must not be too low;

4. in order to appreciate variability in the images induced by a lensing gravitational wave, its period must not be too short, let us say $P<10^{8} \mathrm{~s}$.

5. to ensure that the point where lensing takes place is in the wave zone of the gravitational field, the impact parameter $r$ must obey the condition

$$
r>\lambda_{g w} .
$$

In order to satisfy 1), only electromagnetic radiation with wavelength $\lambda_{e m}$ satisfying $\lambda_{g w} \cdot\left(\lambda_{g w} / D\right)<\lambda_{e m}<\lambda_{g w}$ will be considered. To satisfy 2) note that, if $\delta \sim h$ is the deflection angle, it must be

$$
h \geq 5 \cdot 10^{-9} .
$$

3) depends on the particular processes generating gravitational radiation. Since these are almost all purely speculative, their rates of occurrence are largely or completely unknown and we can only guess their values. A continuous source of gravitational radiation will give rise to a permanent lens, while a gravitational wave burst will constitute a temporary lens.

The sources of gravitational waves that are most often considered in the literature are:

- stellar collapse with non-spherical symmetry;

- formation of massive black holes in active galactic nuclei;

- neutron star collision;

- black hole collision;

- close binary systems;

\footnotetext{
${ }^{4}$ If one is not interested in the possibility of resolving the multiple images created by the gravitational wave, but merely in the occurrence of high amplification events, the limit $10^{-3}$ arcseconds can be considerably relaxed.
} 
- black hole accretion.

The last two types of objects are continuous sources of gravitational radiation, while the others give bursts. In addition, we will consider the stochastic gravitational wave background, both primordial or generated (13, 36, 37] and references therein). Gravitational waves generated by a process involving a body of mass $M$ and size $R$ have dimensionless amplitudes (near the source) of order

$$
h_{S} \sim \epsilon \frac{M}{R}
$$

where the efficiency $\epsilon$ is defined as the fraction of energy radiated away. For processes involving neutron stars or black holes one can assume $M / R \sim 1 / 20$ and $M / R \sim 1$, respectively.

Multiple imaging by gravitational waves is, in principle, possible, and it should be expected if they satisfy the approximate condition (4.12). We examine the astrophysical sources of gravitational radiation which are most often considered in the literature, in conjunction with the condition (4.12). When the event generating gravitational waves involves neutron stars or black holes, the ordinary lensing associated to these objects ("microlensing" [23]) should, in principle, be taken into account: however the separation scale between microimages of a distant source created by a compact object is of order $10^{-6}$ arcseconds, not detectable with present techniques, while the multiple images due to the gravitational wave may be detectable. In addition, the effects induced by gravitational waves vary on a scale different from the typical scale of variation of microlensing, and include a frequency shift $\delta \nu / \nu \sim h$, which is absent in ordinary microlensing (and probably unobservable). However, even in the situations in which lensing by gravitational waves cannot be separated by ordinary microlensing, the former may be dominant, and it is important to study how gravitational waves modify the microlensing phenomenon.

\section{Stellar core collapse}

The research program on collapsing homogeneous ellipsoids by Saenz and Shapiro [38]40] has given the expected maximal efficiences for a "cold" and "hot" equation of state as $\epsilon \sim 10^{-2}$ and $\epsilon \sim 10^{-4}$ respectively, for a spectrum of emitted gravitational radiation broadly peaked between $100 \mathrm{~Hz}$ and $1 \mathrm{KHz}$. Taking the lower value $\epsilon \sim 10^{-4}$ one gets $h_{S} \sim 5 \cdot 10^{-6}$ for the wave amplitudes near the collapsing core and the condition $h \sim h_{S} R_{S} / r>5 \cdot 10^{-9}$ implies $r<10^{3} R_{S} \sim 10^{9} \mathrm{~cm}$. On the other hand it must be 
$r>\lambda_{g w} \simeq 3 \cdot 10^{7}-10^{8} \mathrm{~cm}$; there is a rather narrow permitted range for the impact parameter $\left(r \sim 10^{8} \mathrm{~cm}\right)$, that gives $D h / P \sim 10$ if $D \sim 6 \cdot 10^{15} \mathrm{~cm}$.

If the late phase when the ellipsoid has settled down as a rapidly rotating neutron star is taken into account, it is found 41 that the emitted spectrum is very narrowly peaked $\left(\Delta \nu / \nu \sim 10^{-3}\right)$ and $\epsilon \sim 10^{-6}$ at $\nu \sim 1 \mathrm{KHz}$, that gives $h_{S} \sim 5 \cdot 10^{-8}$. Condition (6.2) requires $r<10 R_{S} \sim 10^{7} \mathrm{~cm}$; on the other hand, condition (6.1) does not allow for lensing on a relevant scale to take place in the wave zone.

If the core keeps bouncing, its eccentricity becomes large after a sufficient number of bounces. This asymmetry 40] makes the efficiency almost uniformly near its maximum value for any initial period above $1 \mathrm{sec}$ to several hundred seconds [42. One gets $h_{S} \sim$ $10^{-2}$ at $\nu \sim 1 \mathrm{KHz}$; the condition (6.2) gives $r<2 \cdot 10^{6} R_{S} \sim 2 \cdot 10^{12} \mathrm{~cm}$, while (6.1) gives $r \geq 3 \cdot 10^{7} \mathrm{~cm}$. A rather large range of values of the impact parameter is permitted; one obtains $D h / P \sim 10$ if $D \sim 10^{12} \mathrm{~cm}, r \sim 3 \cdot 10^{7} \mathrm{~cm}$, or if $D \sim 6 \cdot 10^{16} \mathrm{~cm}, r \sim 2 \cdot 10^{12} \mathrm{~cm}$.

Studies of the perturbations of pressureless spherical collapse leading to the formation of a black hole [43]-45] give results that could possibly be extrapolated to larger deviations from spherical symmetry 42, getting $\epsilon \sim 2 \cdot 10^{-2}\left(\frac{J}{M^{2}}\right)^{4}$ at $\nu \sim$ $1 K H z \cdot\left(M / 10 M_{\odot}\right)^{-1}$ for $J / M^{2} \ll 1$ (where $J$ is the angular momentum and $J=M^{2}$ corresponds to a maximally rotating Kerr black hole). Taking $J \sim 0.1 M^{2}$ and $M=10 M_{\odot}$, it follows that $h_{S} \sim 2 \cdot 10^{-6}$ and (6.2) implies $r<400 R_{S} \sim 4 \cdot 10^{8} \mathrm{~cm}$, while (6.1) gives $r \geq 3 \cdot 10^{7} \mathrm{~cm}$. A rather narrow range of values of $r \sim 10^{8}$ is permitted, that gives $D h / P \sim 10$ if $D \sim 3 \cdot 10^{16} \mathrm{~cm}$.

\section{Final decay of a neutron star/neutron star binary}

Rough estimates for the final decay of a binary system composed of two neutron stars [46] give $\epsilon \sim 5 \cdot 10^{-3}$ at $\nu \leq 2-3 \mathrm{KHz}$. Taking $\nu \sim 500 \mathrm{~Hz}$ it follows that $h_{S} \sim 2.5 \cdot 10^{-4}$. (6.2) gives $r<5 \cdot 10^{4} R_{S} \sim 5 \cdot 10^{10} \mathrm{~cm}$, while $r>6 \cdot 10^{7} \mathrm{~cm}$ due to (6.1). A large range of values of $r$ is permitted; we get $D h / P \sim 10$ if $D \sim 3 \cdot 10^{16} \mathrm{~cm}, r \sim 5 \cdot 10^{10} \mathrm{~cm}$, or if $D \sim 6 \cdot 10^{7} \mathrm{~cm}, r \sim 6 \cdot 10^{7} \mathrm{~cm}$.

\section{Black hole collisions}

The head-on collision of two equally massive, non-rotating black holes has been studied numerically 47], leading to a single, larger, black hole, with efficiency $\epsilon \sim$ $7 \cdot 10^{-4}$. If the two initial black holes have nearly enough angular momentum to go into orbit before coalescing, a formula derived from extrapolation of perturbation theory [42, 47] gives $\epsilon \sim 3 \cdot 10^{-2}$. This efficiency is expected to hold for $P \sim 1 \mathrm{~s}$; (6.2) gives 
$r<6 \cdot 10^{6} R_{S} \sim 6 \cdot 10^{12} \mathrm{~cm}$, while (6.1) implies $r \geq 3 \cdot 10^{10} \mathrm{~cm}$. In the permitted range of values of $r$ the condition $D h / P \sim 10$ is satisfied if $D \sim 3 \cdot 10^{17} \mathrm{~cm}, r \sim 3 \cdot 10^{10} \mathrm{~cm}$ and if $D \sim 6 \cdot 10^{19} \mathrm{~cm}, r \sim 10^{12} \mathrm{~cm}$.

The case of coalescing black holes was studied also in Ref. [22]. Although multiple imaging was not considered in that paper, the deflection angle of light rays was computed using a gauge-invariant formalism.

\section{The binary pulsar}

The binary pulsar PSR 1913+16 (49] and references therein) is believed to radiate gravitational waves in a continuous way, according to the predictions of general relativity ([50 and references therein). The estimated distance of the binary system (believed to be a neutron star/neutron star system) is $D \sim 5 \mathrm{Kpc}$ and the frequency of the radiation is twice the orbital frequency (due to the quadrupole nature of the radiation). From these values one obtains $D / P \sim 3.5 \cdot 10^{7}$ and $D h / P \sim 10$ if $h \sim 3 \cdot 10^{-7}$. An estimate of the amplitude of the waves emitted by the binary pulsar gives

$$
h \sim \frac{\ddot{Q}}{r} \sim \frac{M a^{2} \omega^{2}}{r},
$$

where $Q$ is the quadrupole moment, $M$ is the mass and $a \sim 7 \cdot 10^{10} \mathrm{~cm}$ [50] is the semimajor axis of the binary system, so that $h_{S} \sim(a \omega / c)^{2} \sim 10^{-6}$. It is easy to see that the conditions (6.2) and (6.1) are not compatible. Therefore multiple imaging by the gravitational waves emitted by the binary pulsar PSR $1913+16$ is impossible.

\section{The gravitational wave background}

For the gravitational wave background, both primordial or generated ([13] and references therein), one has

$$
h \sim \sqrt{\Omega_{g w}} \frac{P_{0}}{R}
$$

where $P_{0}$ is the present gravitational wave period, $R$ is the radius of the universe and $\Omega_{g w}$ is the cosmological density of gravitational waves (in units of the critical density). One has

$$
\frac{D h}{P} \sim \sqrt{\Omega_{g w}} \frac{D}{R} .
$$

\footnotetext{
${ }^{5}$ The orbital period is $\sim 2.8 \cdot 10^{4} \mathrm{~s}[50]$.
} 
Upper bounds on $\Omega_{g w}$ have been set in various bands of frequencies. Apart from the obvious bound $\Omega_{g w}<1$ for every region of the spectrum, the limit $\Omega_{g w} \ll 1$ has been established in many frequency bands ([13, 36, 37] and references therein). Moreover, $D / R<1$, hence it is likely that $D h / P \ll 1$ (the exact value depending on the frequency band) and one concludes that multiple imaging by the gravitational wave background is, on average, impossible.

We conclude this section with an example: gravitational waves emitted in a supernova collapse in the Virgo cluster. While the dimensionless amplitude $h_{1} \sim 10^{-21}$ on the earth (at a distance $r_{1} \simeq 15 \mathrm{Mpc}$ ), one has $h_{2} \sim h_{1} r_{1} / r_{2}$ at a distance $r_{2}$. If $r_{2} \sim 1 \mathrm{pc}$,

$h_{2} \sim 1.5 \cdot 10^{-4}$ and assuming $P \simeq 10^{-3} \mathrm{~s}$, Eq. (4.10) tells us that $\sqrt{f(\alpha)} D h_{2} / P$ has to be greater than, or of the order unity in order to have multiple images. This gives $(1-\epsilon)^{-1} \leq D_{S} / D_{L S} \leq \epsilon^{-1}$, where $\epsilon=4.4 \cdot 10^{-15}$, hence multiple imaging is possible with a light source located virtually anywhere beyond the Virgo cluster (of course this situation is different if one changes the parameters $h_{2}, P$ ).

The amplification on a caustic is infinite in the geometric optics approximation and is limited by geometric optics. For ordinary gravitational lenses, $\mathcal{A} \sim\left(M / \lambda_{e . m}\right)^{1 / 3}$ on a fold caustic and $\mathcal{A} \sim\left(M / \lambda_{\text {e.m. }}\right)^{1 / 2}$ on a cusp caustic, where $M$ is the mass of the lens and $\lambda_{e . m}$. is the electromagnetic wavelength [16]. Naively, one expects a similar result for lensing gravitational waves, when $M$ is substituted with $(h / P)^{2} l^{3}$, where $l$ is the size of the gravitational wave packet. However, a realistic computation on the lines of [23] is much more complicated and requires the use of precise models that we have not considered here.

\section{$7 \quad$ Discussion and conclusions}

Gravitational waves affect the propagation of light; this is expected for any gravitational field. The interaction between light and gravitational waves in general relativity has been considered by many authors [2]-[12]. Recently, the attention was focussed upon the contribution of gravitational waves to the microwave background anisotropies discovered by the $C O B E$ experiment [14, and on the possibility of detecting astrophysically generated (as opposed to primordial) gravitational waves through their effects on light rays [19]-22]. The possibility of gravitational waves superimposed to an ordinary gravitational lens was also considered [16]-18]. The generalization of the Fermat principle to non-stationary spacetimes [30] allows one to approach the problem using a modified version of the vector formalism employed for ordinary gravitational lenses [29]. An ap- 
proximate condition (Eq. (4.12)) analogous to that holding for ordinary gravitational lenses must be satisfied in order to create multiple images of a distant light source. The astrophysical sources of gravitational waves most often considered in the literature have been examined. Certain astrophysical sources are shown to satisfy the approximate condition for the creation of multiple images, due to the balance between the large values of the distances involved and the small values of the gravitational wave amplitudes. Another relevant fact is that lensing can take place in regions relatively close to the sources of gravitational waves, where the wave amplitudes are larger than those expected in the Solar System. The gravitational wave background, instead, is unlikely to produce multiple images. As a conclusion, multiple imaging of a distant source by gravitational waves is possible in the favourable situations examined, including the collapse of stellar cores, the final decay of neutron star/neutron star binaries and black hole collisions. Unfortunately, these events (in particular black hole collisions) are very rare and the probability of observing the phenomenon is extremely low, mainly because the duration of the multiple images is limited to the period of intense emission of gravitational radiation. Continuous sources of gravitational waves like the binary pulsar PSR 1913+16 would give a much higher probability, but unfortunately they emit gravitational waves too weakly.

It is to be noted that the limit $\delta \geq 10^{-3}$ arcseconds in condition 2) of Sec. 6 can be considerably relaxed if we do not require that the multiple images created by gravitational waves be resolved. The high amplification events associated to the presence of multiple images would still constitute an interesting and observable phenomenon even if the multiple images are not resolved. In fact, this situation occurs in ordinary microlensing by stars or planets: the typical scale of separation of microimages is $10^{-6}$ arcseconds or less, yet the phenomenon has been observed [51, 52]. Moreover, it was not necessary to consider cosmological distances $D$ in order to have an appreciable probability for microlensing: the phenomenon was observed in the Large Magellanic Cloud [51] and in our galaxy [52.

Further work is necessary to assess precisely the probability of observing multiple images created by gravitational waves from astrophysical sources, and the details of the phenomenon. Contrarily to the conclusions of Ref. [19, we are not very optimistic on the probability of observing such an event, but our results show that this possibility deserves some attention. In addition, we restricted our attention to the creation of multiple images, while Ref. [19] include other aspects of lensing. It is also useful to remember that, before the discovery of the first gravitational lens system in 1979, gravitational lenses were considered mere speculations not occurring in the real world. Today, not only the existence of gravitational lenses is universally acknowledged, but gravitational 
lensing has become one of the most active and promising fields of astronomy.

\section{Acknowledgments}

The author is deeply indebted to B. Bertotti for suggesting the possibility of lensing by gravitational waves and the approach used in this paper, and for helpful discussions. Thanks are due also to G.F.R. Ellis for stimulating discussions and to a referee for suggestions leading to improvements in the manuscript.

\section{Appendix}

Here we provide an independent proof of the equation $\partial_{A}\left(\delta p^{A}\right)=0$, which was found also in Ref. [25]. From Eq. (2.2) and from the normalization $p_{\mu} p^{\mu}=0$ we obtain, to first order,

$$
2 \eta_{\mu \nu} p_{(0)}^{\mu} \delta p^{\nu}+h_{\mu \nu} p_{(0)}^{\mu} p_{(0)}^{\nu}=0
$$

and

$$
\delta p^{0}-\delta p^{3}=\frac{1}{2}\left(h_{00}+2 h_{03}+h_{33}\right)+\mathrm{O}\left(h^{2}\right) .
$$

From Eq. (2.12) it follows that $\partial_{3}\left(\delta p^{\mu}\right)=0$ and Eq. (A.2) gives

$$
\partial_{3}\left(h_{00}+2 h_{03}+h_{33}\right)=0+\mathrm{O}\left(h^{2}\right) \text {. }
$$

Equation (2.12) gives $\delta p^{3}=0$ and from Eq. (A.2) it follows that

$$
\delta p^{0}=\frac{1}{2}\left(h_{00}+2 h_{03}+h_{33}\right)+\mathrm{O}\left(h^{2}\right) .
$$

By setting $f \equiv\left(h_{00}+2 h_{03}+h_{33}\right) / 2$, it follows from Eq. (2.12) that $\partial_{3} f=-\partial_{0} f$ and

$$
\partial_{00}^{2} f-\partial_{33}^{2} f=0
$$

along the photon trajectory. Using the linearized Einstein equations for gravitational waves propagating outside their sources,

$$
\begin{gathered}
\square \bar{h}_{\mu \nu}=0, \\
\partial^{\nu} \bar{h}_{\mu \nu}=0,
\end{gathered}
$$


where $\bar{h}_{\mu \nu} \equiv h_{\mu \nu}-\eta_{\mu \nu} h / 2$, one obtains

$$
\square h_{00}=-\square h_{33}=-\frac{1}{2} \square h \quad, \quad \square h_{03}=0 .
$$

From Eqs. (A.8) one obtains

$$
\square f=0
$$

and

$$
\partial_{\mu}\left(\delta p^{\mu}\right)=\frac{1}{2} \int_{S}^{O} d z\left(h_{00}+2 h_{03}+h_{33}\right)_{, \mu}^{, \mu}=0+\mathrm{O}\left(h^{2}\right) .
$$

The two-dimensional divergence $\partial_{A}\left(\delta p^{A}\right)$ is given by

$$
\partial_{A}\left(\delta p^{A}\right)=\int_{S}^{O} d z f_{, A}^{, A}=\int_{S}^{O} d z\left(\square f+\frac{\partial^{2} f}{\partial t^{2}}-\frac{\partial^{2} f}{\partial z^{2}}\right)=0,
$$

where the last equality follows from Eqs. (A.5) and (A.9). 


\section{References}

[1] H. Bondi, F.A.E. Pirani and I. Robinson, Proc. R. Soc. Lon. A 251, 519 (1959); R. Penrose, Rev. Mod. Phys. 37, 215 (1965); in Perspectives in Geometry and Relativity: Essays in Honor of Vàclav Hlavatý, ed. by B. Hoffman (Indiana Univ. Press, Bloomington, 1966); H. Bondi and F. A. E. Pirani, Proc. R. Soc. Lond. A 421, 395 (1989).

[2] D. M. Zipoy, Phys. Rev. 142, 825 (1966).

[3] F. Winterberg, Nuovo Cimento 53B, 1096 (1968).

[4] D. M. Zipoy and B. Bertotti, Nuovo Cimento 56B, 195 (1968).

[5] P. G. Bergmann, Phys. Rev. Lett. 26, 1398 (1971).

[6] G. Dautcourt, Mon. Not. Roy. Astr. Soc. 144, 255 (1969); in Confrontation of Cosmological Theories with Observation, IAU Symposium No. 63, ed. by M. S. Longair (Reidel, Dordrecht, 1974); Astron. Astrophys. 38, 344 (1975); Astron. Nachr. 298, 81 (1977).

[7] M. J. Rees, Mon. Not. Roy. Astr. Soc. 154, 187 (1971).

[8] B. Bertotti and R. Catenacci, Gen. Rel. Grav. 6, 329 (1975).

[9] W. L. Burke, Astrophys. J. 196, 329 (1981).

[10] V. Faraoni, Astron. Astrophys. 272, 385 (1993).

[11] E. V. Linder, Phys. Rev. D 34, 1759 (1986).

[12] V. B. Braginsky et al., Nuovo Cimento 105B, 1141 (1990).

[13] B. J. Carr, Astron. Astrophys. 89, 6 (1980).

[14] R. K. Sachs and A. M. Wolfe, Astrophys. J. 147, 73 (1967); A. V. Korotun, Sov. Astron. 24, 273 (1979); Ya. B. Zel'dovich and I. D. Novikov, Relativistic Astrophysics, Vol. 2. The Structure and Evolution of the Universe (University of Chicago Press, Chicago, 1983); A. G. Polnarev, Sov. Astron. 29, 607 (1985); A. A. Starobinsky, Sov. Astron. Lett. 11, 133 (1985).

[15] A. R. Liddle and D. H. Lyth, Phys. Rep. 231, 1 (1993); and references therein.

[16] B. Mc Breen and L. Metcalfe, Nature 332, 234 (1988).

[17] B. Allen, Phys. Rev. Lett. 63, 2017 (1989); Gen. Rel. Grav. 22, 1447 (1990). 
[18] J. A. Frieman, D. D. Harari and G. C. Surpi, Phys. Rev. D 50, 4895 (1994).

[19] A. Labeyrie, Astron. Astrophys. 268, 823 (1993).

[20] R. Fakir, Astrophys. J. 418, 202 (1993).

[21] R. Fakir, preprint UBCTP-93-016 (1993); Phys. Rev. D 50, 3795 (1994); Astrophys. J. 426, 74 (1994); preprint UBCTP-94-011 (1994); preprint astro-ph 9507112 (1995).

[22] R. Durrer, Phys. Rev. Lett. 72, 3301 (1994).

[23] P. Schneider, J. Ehlers and E. E. Falco, Gravitational Lenses (Springer-Verlag, New York, 1992).

[24] J. A. Wheeler, in Rendiconti della Società Italiana di Fisica, 11th Course of the Varenna Summer School (Academic Press, New York, 1961) p. 67.

[25] B. Bertotti, in General Relativity and Cosmology, ed. by R. K. Sachs (Academic Press, New York, 1971).

[26] B. Bertotti, private communication.

[27] V. Faraoni, in Gravitational Lenses, Proceedings, Hamburg 1991, ed. by R. Kayser, T. Schramm and L. Nieser (Springer-Verlag, Berlin, 1992); in Proceedings of the Pacific Conference on Gravitation and Cosmology, Seoul, Korea 1996 (astro-ph/9610164), in press.

[28] R. D. Blandford and C. S. Kochanek, in Proceedings of the 13th Jerusalem Winter School on Dark Matter in the Universe, ed. by J. N. Bachall, T. Piran and S. Weinberg (World Scientific, Singapore, 1988).

[29] V. Faraoni, Astrophys. J. 398, 425 (1992).

[30] I. Kovner, Astrophys. J. 351, 114 (1990); V. Perlick, Class. Quant. Grav. 7, 1319 (1990); R. Nityananda and J. Samuel, Phys. Rev. D 45, 3862 (1992).

[31] K. Subramanian and S. A. Cowling, Mon. Not. Roy. Astr. Soc. 219, 333 (1986).

[32] I. M. H. Etherington, Phil. Mag. Ser. 7 15, 761 (1933).

[33] E. L. Turner et al., Astrophys. J. 284, 1 (1984).

[34] V. Faraoni, Phys. Lett. 153A, 67 (1991).

[35] W. L. Burke, Astrophys. J. (Lett.) 244, L1 (1981). 
[36] L. A. Rosi and R. L. Zimmerman, Astrophys. Space Sci. 45, 447 (1976).

[37] R. A. Matzner, preprint (1988).

[38] R. A. Saenz and S. L. Shapiro, Astrophys. J. 221, 286 (1978).

[39] R. A. Saenz and S. L. Shapiro, Astrophys. J. 229, 1107 (1979).

[40] R. A. Saenz and S. L. Shapiro, Astrophys. J. 244, 1033 (1981).

[41] S. Detweiler and L. Lindblom, Astrophys. J. 250, 739 (1981).

[42] D. M. Eardley, in Gravitational Radiation, ed. by N. Deruelle and T. Piran (North Holland, Amsterdam, 1983).

[43] V. Moncrief et al., in Sources of Gravitational Radiation, ed. by L. L. Smarr (Cambridge University Press, Cambridge, 1979).

[44] C. T. Cunningham et al., Astrophys. J. 224, 643 (1978).

[45] C. T. Cunningham et al., Astrophys. J. 230, 870 ( 1979).

[46] J. P. Clark and D. M. Eardley, Astrophys. J. 215, 311 (1977).

[47] L. L. Smarr, in Sources of Gravitational Radiation, ed. by L. L. Smarr (Cambridge University Press, Cambridge, 1979).

[48] J. P. Clark, ibidem.

[49] R. A. Hulse, Rev. Mod. Phys. 66, 699 (1994); J. H. Taylor Jr., ibidem, 711.

[50] C. M. Will, Theory and Experiment in Gravitational Physics, revised edition (Cambridge University Press, Cambridge, 1993).

[51] E. Aubourg et al., Nature 365, 623 (1993).

[52] A. Udalski et al., Acta Astr. 43, 289 (1993). 